Revue internationale P.M.E.

Économie et gestion de la petite et moyenne entreprise

\title{
L’entrepreneuriat : une vue kaléidoscopique
}

\section{Michel Marchesnay}

Volume 13, numéro 1, 2000

URI : https://id.erudit.org/iderudit/1008672ar

DOI : https://doi.org/10.7202/1008672ar

Aller au sommaire du numéro

Éditeur(s)

Presses de l’Université du Québec

ISSN

0776-5436 (imprimé)

1918-9699 (numérique)

Découvrir la revue

Citer cette note

Marchesnay, M. (2000). L'entrepreneuriat : une vue kaléidoscopique. Revue internationale P.M.E., 13(1), 105-116. https://doi.org/10.7202/1008672ar d'utilisation que vous pouvez consulter en ligne.

https://apropos.erudit.org/fr/usagers/politique-dutilisation/ 


\section{Note de lecture}

\section{L'entrepreneuriat: une vue kaléidoscopique}

Michel MARCHESNAY

Université de Montpellier I

Il est banal de constater que l'entrepreneuriat est devenu à la mode - du moins en France, puisque, dans les pays anglo-saxons, fondés sur une économie d'entreprises, l'entrepreneuriat est ancré dans les gènes. Il est également de bon ton de déplorer l'absence d'esprit d'entreprise dans l'Hexagone, surtout chez certains auteurs nordaméricains : il est vrai que, lorsqu'on apprend (Le Monde, 18 juin 1999) que, à l'ENA «les disciplines dites managériales [sic] font l'objet d'un enseignement marginal - deux semaines sur une année » et qu' "elles sont optionnelles jusqu'en 1998 », il y a de quoi s'inquiéter sur la formation des futurs dirigeants d'entreprises françaises!

Cet engouement, somme toute tardif, est-il sincère ? Comme on le constate avec la création - réussie - de l'Académie de l'entrepreneuriat, les établissements d'enseignement (écoles de commerce et d'ingénieurs, universités) semblent se faire les prosélytes de ce fameux «entrepreneuriat » (encore appelé «entreprenariat» par l'OCDE). Mais est-on sûr que tout le monde parle de la même chose?

La réponse la plus immédiate, et la plus concrète, est donnée par la lecture de textes qui évoquent, peu ou prou, ce nouvel état d'esprit, ou cette nouvelle fonction de, ou dans l'entreprise. Il va de soi que la sortie annoncée de la Société salariale (qui reste d'ailleurs à démontrer) met en avant la nécessité de «créer son propre emploi », et, partant, de devenir entrepreneur en « se mettant à son compte ». De façon plus générale, 1 ' « esprit du temps » favoriserait un certain «nomadisme ». Déjà, dans Mille plateaux, en 1980, Deleuze et Guattari (1997), énoncent un «traité de nomadologie »; à l'appareil d'État (et plus généralement, aux organisations structurantes, comme la bureaucratie managériale) s'opposerait une «machine de guerre »- entendons des entités manœuvrant dans des espaces «lisses» (et non «striés ») de façon agressive. Or, n'oublions pas que Cantillon met dans le même sac les titulaires de revenus à "gage incertain » (l'entrepreneur va du négociant à la prostituée), que Veblen vilipende les «barons pillards », que Casson, plus près de nous, prend comme exemple d'entrepreneurs les trafiquants de drogue... Plus récemment, Michel Maffesoli, traitant du nomadisme, envisage l'homme postmoderne comme un « rebelle» combinant l'affect et les technologies les plus 
avancées. Or, dit-il, en rappelant le rôle de l' « errance » dans le capitalisme étudié par Braudel, «le marché est toujours par excellence le lieu de l'effervescence» (1997, p. 53). Plus loin (p. 132), il évoque implicitement le «nouvel esprit d'entreprise » postmoderne : « sédentaires sans patrie, aventuriers immobiles, tels que [sic] furent certains esprits d'élite communiant, de leur lieu, à des cultures diverses. Tels sont certainement [re-sic], nouvelles technologies aidant, les chercheurs du Graal contemporains ».

À cette aune, le risque de logomachie devient évident. On peut alors espérer que les travaux des spécialistes vont nous rassurer, en s'accordant sur une « convention d'entrepreneur ». Force est de constater qu'il n'en est rien. La polysémie ambiante tient, à notre sens, à deux données différentes :

- dès l'abord, les définitions de l'entrepreneur incluent, dans l'histoire des idées, des attributs variés dont chacun peut donner lieu à une « théorie sur l'entrepreneur».

- mais, de nos jours, de nouveaux arrangements se font jour, de nouvelles formes entrepreneuriales surgissent, aux limites de l'oxymoron, à savoir des définitions rassemblant des attributs contradictoires (l'«obscure clarté»).

Une évocation superficielle de travaux récents nous convainc de cette impression de kaléidoscope, pour reprendre une expression utilisée par Fritz Machlup en son temps.

\section{Les thèmes récurrents de l'entrepreneuriat}

Les discours sur l'entrepreneuriat introduisent des leitmotivs, avec des intensités variables.

Dans ce que nous appellerons l' «entrepreneuriat pur », les thèmes - les piliers - sont : le risque assumé, l'incertitude créée et exploitée, le profit qui en est tiré. De ces piliers sont déduites les «fonctions » inhérentes à l'esprit d'entreprise : la création, l'innovation et la rentabilisation. Ces fonctions s'appuient sur des aptitudes, des «capacités » : la créativité, l'innovativité et la profitabilité.

Sur cette base se sont établies des représentations de l'«entrepreneur », largement fondées sur des métaphores : le chevalier (en quête d'un « graal » quelconque), le guerrier-stratège, l'aventurier, le nomade, voire le prophète-visionnaire. On retrouve ici des expressions que nous avons précédemment évoquées.

Cette conception «héroïque » de l'entrepreneur trouve un écho dans les discours actuels. Dans cette veine, on ne saurait trop recommander la lecture d'un excellent petit ouvrage consacré au « risque d'entreprendre », paru dans la collection «Les Essentiels» aux Éditions Milan. Réalisé par un collectif, Polynome, de 
professionnels du conseil, cet opuscule tient la gageure de mettre en valeur tous les aspects des risques pris par l'entrepreneur, en adoptant une vision « distanciée », non dénuée d'humour. Les auteurs soulignent combien le «risque», qu'ils définissent avec sagacité, est inhérent à la volonté d'entreprendre, au sens large du terme. Il y a là une excellente initiation à l'entrepreneuriat, à côté de présentations plus pédagogiques.

Dans cette dernière perspective, on citera en premier lieu le récent ouvrage de Boutillier et Uzunidis (1999), consacré à «la légende de l'entrepreneur ». Notamment dans la première partie, les auteurs exposent les diverses acceptions, autant de l'entrepreneur que de l'esprit d'entreprise, dans l'histoire de la pensée économique, quitte à montrer ambiguïtés et contradictions. Ils soulignent le rôle du «capital social», fait à la fois de légitimité, d'intégration sociale et territoriale - encore que le risque soit grand, dans cette acception, de sortir de l' «entrepreneuriat pur » pour évoquer plutôt le chef d'entreprise. En outre, au même titre que le concept de «milieu novateur» est de plus en plus soumis à un examen critique, la notion de «milieu innovateur » l'est aussi, tandis que celle de «milieu entreprenant » se heurte à des objections croissantes.

On aurait garde d'oublier l'excellente synthèse sur les théories de l'entrepreneur dans l'ouvrage de Robert Wtterwulghe (1999) consacré à la PME, dans le chapitre 2.

Mais il ressort de ces travaux le sentiment d'une confusion croissante entre l'« entrepreneur et l'entrepreneuriat purs » et l' « entrepreneur phénoménal », historiquement, socialement, institutionnellement positionné. Dans ce cas, il vaut mieux parler de «chef d'entreprise », de " patron », voire de « dirigeant » ou d' « artisan », dans la mesure où ces personnes n'assument que partiellement les fonctions précitées et ne possèdent que partiellement les aptitudes requises.

Au demeurant, la confusion vient de Say, chef d'entreprise, qui privilégie les actes de gestion, alors que l'entrepreneur «pur » est à notre sens un « stratège pur ». Charles Gide fera bien la distinction, en accordant une place particulière au «patronat».

Dans cette acception, il était logique que l'économie pure fasse disparaître le décideur d'entreprise de la théorie de l'équilibre général de marché... et que Schumpeter réintroduise l' « entrepreneur » dans la dynamique, à travers une « fonction entrepreneuriale », combinant l'innovateur (Watt) et le stratège (Bolton) pour proposer de nouvelles combinaisons de production.

Ces brèves remarques avaient pour but de nous aider à mieux comprendre en quoi les publications récentes consacrées à notre sujet recèlent des contradictions, des imprécisions, voire des amphigouris apparents, mais aussi combien elles révèlent un élargissement de l'acte d'entreprendre dans une société «nomadisée » et anomique. 


\section{L'entrepreneur comme créateur d'entreprise}

Dans les discours actuels, l'entrepreneur est largement assimilé au créateur de « son » entreprise. L'argument repose sur la nécessité de résorber le chômage et de créer les « emplois de demain », qui devraient émerger d'une sorte de prolifération d'entreprises, dont un grand nombre seront appelées à disparaître, mais dont certaines feront souche et grandiront, devenant de « vraies » entreprises, à l'instar de certaines sagas dignes des « soap operas »... Il est vrai que la situation peut paraître préoccupante, dans la mesure où le nombre de créations nettes ne cesse de décroître en France.

Toutefois, ce discours alarmiste peut être tempéré par l'importance croissante des reprises d'entreprises viables, par les héritiers ou par des repreneurs, issues du baby-boom et de la croissance des 50 dernières années.

Il n'en reste pas moins que nombre de publications abordent cette question de la création : il suffit de jeter un regard sur les éventaires des kiosques de gare !

Particulièrement représentatif de cette focalisation sur la création nous paraît être l'ouvrage d'Émile-Michel Hernandez (1999), par ailleurs auteur de plusieurs articles dans cette revue. Son ouvrage, paru chez L'Harmattan, est consacré au « processus entrepreneurial »; celui-ci est largement assimilé à l'acte de création d'entreprise, dans le lignée de chercheurs comme Christian Bruyat ou Guy Pendeliau (on notera une excellente bibliographie en fin d'ouvrage).

L'entrepreneur est vu avant tout comme un initiateur, possédant des capacités spécifiques (traits de personnalité, etc.) et développant une stratégie de mise en œuvre d'une idée dans une nouvelle entreprise. Cette stratégie est largement assimilée à la mise en œuvre d'un projet autour de points clés (buts, organisation, activités, environnement).

Dans la même veine, toujours chez L'Harmattan, Sylvie Sammut évoque la phase de démarrage, jugée essentielle pour la survie de la « jeune entreprise » (titre de l'ouvrage). On retrouve la même approche autour des quatre «piliers » du système de gestion. Le rôle crucial du «dirigeant» est mis en valeur : ainsi, une fois passée la phase de création, l' «entrepreneur» se mue en «gestionnaire » et consolide son affaire en « exploitant», dans les divers sens du terme, «son » idée, dans « son » entreprise. Dans cette optique, l'esprit d'entreprise serait donc lié avant tout à la gestation puis à la gestion de l'idée à la source de la création.

L'ouvrage publié par l'OCDE propose des recommandations aux États membres pour «stimuler l'esprit d'entreprise». On ne sera pas étonné du ton diplomatique employé dans ce type de publications : toutefois, les experts prônent le développement d'une économie d'entreprises, fondée sur le libre fonctionnement des marchés, sur la déréglementation, sur un soutien logistique de la puissance 
publique. Les experts notent également que l' «esprit d'entreprise » (distingué de l'entrepreneur proprement dit, en tant que propriétaire-dirigeant) est lié à des conditions socioculturelles. Ils font observer en conséquence que des dirigeants non propriétaires peuvent être «entreprenants » (ce qui n'est pas nécessairement le cas de chefs d'entreprise), mais aussi que l'esprit d'entreprise peut apparaître dans des projets socioculturels, qu'ils soient dans le secteur marchand ou non ; on reviendra sur ce point plus loin.

\section{L'entrepreneur-manageur}

Accoler ces deux termes constituerait, aux yeux de certains, un oxymoron, dans la mesure où ils semblent s'opposer. En effet, le manageur n'est pas propriétaire et n'assume donc pas le risque entrepreneurial sur son capital, voire sur son patrimoine : il est un simple agent.

La question s'éclaire si, comme on vient de le rappeler, on distingue l' «entrepreneur », propriétaire-dirigeant, individuel ou collectif (familial par exemple, comme dans le "système Motte», évoqué par Piore et Sabel, aujourd'hui «le système Mulliez ») et l' « esprit d'entreprise ». Ainsi, peut-on distinguer, dans les PME et les TPE, un état d'esprit « de survie » (entrepreneur isolé), « de légitimité » (notable, patron), « de professionnalisme» (nomade, manageur, PDG) et enfin «d'entreprise » (innovativité, prise de risque, etc. : soit l'entreprenant).

Telle est bien la question que pose Olivier Torrès dans un petit ouvrage de la collection «Domino » (Flammarion), lorsqu'il évoque la « dénaturation » de la PME. Refusant le dilemme entre une conception anamorphique ou métamorphique des organisations d'entreprises, l'auteur réfute l'idée d'une spécificité de la PME, par rapport à la grande entreprise, et montre que certaines, parfois très petites, sont gérées «comme des grandes ». Cette thèse, qui a donné lieu à plusieurs contributions dans cette revue, nous semble surtout confirmer le rôle auto-organisant de l'entrepreneur. On peut penser que les PME organisées de façon «managériale » le sont par des dirigeants (seuls ou collectifs) ayant œuvré au préalable dans des bureaucraties professionnelles (comme cadre, ingénieur, voire dirigeant). Mais l'on ne saurait croire que l'auteur s'attache à une conception fixiste (à la Geoffroy SaintHilaire) des organisations, en fonction de leur taille... ni qu'il l'attribue aux autres chercheurs en PME ! Bien plutôt, force est de reconnaître le rôle déterminant du transformisme (à la Cuvier) et, surtout, de l'évolutionniste (entre Lamarck et Darwin).

Ainsi, certains auteurs ont-ils mis l'accent sur l'influence déterminante de l'esprit entrepreneurial sur le processus organisationnel. On citera deux publications récentes à titre d'exemple. Thierry Verstraete (auteur de plusieurs contributions dans cette revue) expose avec clarté cette thèse dans un article de la Revue internationale de systémique. Les effets structurants sur l'organisation « de type entrepreneurial » 
(c'est-à-dire gérée par le propriétaire-dirigeant) portent sur trois dimensions : cognitive (apprentissage, vision), praxéologique (positionnement stratégique) et structurale (organisation formelle et informelle). L'approche est ici largement de type sociologique, bien qu'elle rejoigne la préoccupation des économistes de la firme et de l'industrie. Dans un numéro spécial de la Revue d'économie industrielle consacré à l'économie de la connaissance, une contribution traite de l' «autoorganisation et connaissance dans la firme » (Ngo-Mai et Rocchia). Les auteurs soulignent (p. 249) que « curieusement peut-être [sic] l'approche évolutionniste de la firme a accordé peu d'importance à la figure de l'entrepreneur ou [re-sic] à la fonction entrepreneuriale». À leurs yeux, «c'est pour imprimer sa conception des affaires, ou le produit de son imagination qu'un entrepreneur développe une organisation $[. .$.$] La fonction entrepreneuriale consiste donc ici à construire une vision$ partagée des affaires, ou, pour reprendre notre terminologie, à faire partager des représentations types à des agents réceptifs ».

Cette conception affichée du rôle de l'entrepreneuriat rejoint en fait les idées développées par les tenants d'une reconstruction des bureaucraties professionnelles, gérées de façon managériale. L'ouvrage le plus typique de cette tendance est indubitablement celui de Ghoshal et Bartlett. Les auteurs, enseignants-chercheurs à l'INSEAD de Fontainebleau, ont mené une enquête dans de grandes multinationales ; ils en tirent des conclusions sur ce qu'ils appellent l' « entreprise individualisée. Une nouvelle logique de management». L'idée fondamentale est que la grande entreprise doit responsabiliser l'homme de l'organisation. Il s'agit de développer l'autodiscipline (voire l'auto-évaluation), et de «libérer les entrepreneurs », c'est-à-dire le potentiel d'initiative et de créativité, censé habiter chaque salarié « compétent». Reprenant la notion d' "entreprise apprenante » (et «entreprenante»), les auteurs insistent sur la nécessité de faciliter les processus d'adaptation et d'évolution, en développant les compétences des individus - ce qui implique une transformation du rôle du management. Or, cette évolution passe largement par une descente du «processus entrepreneurial » à l'échelon de ce qui était jusqu'ici l'exécution.

Cet ouvrage reprend donc les thèmes à la mode du «nouveau management ». Il est symptomatique que l' ' esprit d'entreprise » y soit magnifié, sans bien le définir au demeurant, comme certains auteurs évoquent le «consommateur-entrepreneur » (de Rochefort). On retrouve des idées dans l'air du temps, sur le besoin d'autonomie et de responsabilisation, d'autocontrôle, de «nomadisme», au sens de Maffesoli.

Il va de soi qu'une telle approche n'est pas dénuée de dangers. Ainsi, JeanPierre Le Goff, dans un ouvrage récent paru à La Découverte, évoque la «barbarie douce » qui se développe à travers la «modernisation aveugle des entreprises et de l'école». Dans les entreprises comme à la maternelle, il s'agit d'inculquer la notion de libre arbitre, d'autocontrôle, d'initiative, toutes attitudes attribuées à l'esprit d'entreprise. L'auteur se livre à une sévère critique de l'ouvrage de Ghoshal et 
Bartlett. Il montre (p. 21) que, sous couvert d'autonomisation « chaque salarié est placé devant une situation contradictoire, profondément déstabilisatrice : il est sommé d'être autonome en même temps qu'il doit se conformer à des normes strictes de performances; il est censé décider en toute autonomie en même temps qu'on lui fait savoir qu'il n'a guère le choix... Dans cette logique, l'échec n'est pas de mise... Les plus faibles sont les premiers fragilisés et angoissés ».

En fait, la question centrale est bien de savoir si la notion de «managemententrepreneuriat » (en y incluant l' « intrapreneuriat », voire l' « extrapreneuriat») a bien un sens : oxymoron sans nul doute, ou tout simplement aporie. Le management, c'est l'art d'éviter les risques en établissant des procédures, alors que l'entrepreneuriat, c'est l'art d'assumer les risques en développant des processus: si les deux modes peuvent cohabiter, leur intégration semble bien problématique...

\section{L'ingénieur entrepreneur}

Aux yeux des Anglo-Saxons, la France apparaît comme un pays dominé par la technocratie, véritable doctrine qui aurait été énoncée par Saint-Simon. Ainsi, les ingénieurs auraient pour tâche de rationaliser la production, de promouvoir la logique industrielle - idée que reprendront en fin de siècle Taylor, Fayol, mais aussi Veblen qui, dans l' «Ère des Ingénieurs », distingue les «businessmen », vautours acharnés à la spéculation financière, des «capitaines d'industrie », annonciateurs des manageurs.

Un «Que sais-je ? consacré à Saint-Simon et au saint-simonisme vient opportunément rectifier la vision de ce personnage, au point que l'on peut se demander si, en fin de compte, Saint-Simon était bien saint-simonien! En effet, il est intéressant de constater que notre auteur a débuté comme aventurier, à la Beaumarchais, officier enrôlé dans la guerre d'Indépendance, puis entrepreneur florissant, manquant d'être guillotiné sous la Terreur - un vrai personnage de feuilleton télévisé ! Par la suite, il se consacre à ses recherches sur l'industrialisme et sur le pouvoir de l'ingénieur. On connait ses libelles, qui lui valent quelques ennuis sous la Restauration; on connaît moins ses travaux sur le rôle des réseaux et des systèmes de communication et d'information, véritable obsession de nos « grands ingénieurs» (X-Mines, X-Ponts, Supélec et Centrale, etc.), adeptes des «Grands Travaux $» \ldots$

Dans cette « Société d'ingénieurs », où le management entre difficilement et marginalement dans l'enseignement des écoles, vouloir développer l'esprit d'entreprise ressemble au pari impossible. Pourtant, un rapport a été remis au secrétaire d'État à l'Industrie sur « la formation entrepreneuriale des ingénieurs ». L'idée générale est de favoriser la création d'entreprise par de jeunes ingénieurs ayant un projet innovant. Encore une fois, le système français privilégie la « culture sous serre », avec incubateur et autres «nurseries », à la mise en concurrence, au 
sein de parcs, bassins, districts, etc. Or, on sait que, plus un projet est innovant, plus l'espérance de survie de la jeune entreprise est faible. Par ailleurs, le problème réside moins dans l'élaboration du projet que dans la «mise à l'eau », au cours des premières années de démarrage, comme l'a montré notamment Sylvie Sammut.

Il faut noter que les rapporteurs ne se font guère d'illusions : les cours d'entrepreneuriat ne concerneraient qu' un nombre fort restreint d'élèves-ingénieurs (à tout casser, un dizième de la promotion, et sans doute moins, comme nous l'avons vérifié personnellement). Par ailleurs, les auteurs observent pertinemment qu' «il faut maintenant admettre que l'on dispose d'un corpus de doctrines qui permet de bâtir un enseignement cohérent : qu'il existe un certain nombre de techniques entrepreneuriales [sic] qui méritent d'être apprises dès l'école et qu'il serait dommage de se contenter de dire qu'elles seront assimilées plus tard en fonction des besoins propres et concrets de chaque créateur lorsqu'il sera question de créer. En conclusion, les programmes de projets seront évidemment [re-sic] prédominants dans la formation optionnelle, mais ce serait une erreur de ne pas les accompagner par une formation théorique».

Ce texte appelle plusieurs commentaires. En premier lieu, l'entrepreneur est assimilé à la création; alors l'âge «idéal » se situe entre 35 et 40 ans. En deuxième lieu, le problème est ramené à l'acquisition de «techniques", alors que l'entrepreneuriat implique avant tout un élan de l'esprit (mais peut-on changer la Société ingéniorale par décret?). En troisième lieu, le rationalisme ambiant conduit à distinguer la «théorie » et la " pratique », comme le potache qui rédige un (mauvais) mémoire de maîtrise, alors que la visée est avant tout praxéologique.

Le rapport constitue néanmoins une avancée significative ; celle-ci est encore plus nette dans l'ouvrage d'Alain Fayolle consacré à «l'ingénieur entrepreneur français », paru à L'Harmattan. L'ouvrage se veut « une contribution à la compréhension des comportements de création et reprise d'entreprise des ingénieurs diplômés ». L'auteur rappelle d'emblée que, parmi les entrepreneurs, 1 à $2 \%$ ont une formation d'ingénieur et que, parmi ceux-ci, environ $7 \%$ en moyenne créent ou reprennent une entreprise. En fait, la question cruciale est de savoir, à notre sens, dans quelle mesure la formation d'ingénieur introduit une spécificité dans le comportement d'entrepreneur, ou si, au contraire, c'est la spécificité « entrepreneuriale » qui l'emporte, à l'instar des questions que l'on se pose sur l'entrepreneuse. Les réponses que fournit Alain Fayolle, à partir d'un dépouillement de quelque 650 réponses à une enquête postale, sont à cet égard partagées, si riches d'enseignements soient-elles. D'un côté, les mobiles qui conduisent à «se jeter à l'eau » sont tout à fait ceux que l'on retrouve dans les travaux sur les créateurs (besoins d'auto-nomie, etc.); d'un autre côté, l'auteur fait apparaître que la population se divise en «entrepreneurs manageurs» et «entrepreneurs techniciens, voire supertechniciens », en fonction, et de leur formation de base, et de leur parcours professionnel. 
Il convient également de noter que, de façon croissante, les restructurations des grandes bureaucraties professionnelles, avec leurs « charrettes », vont conduire les ingénieurs et techniciens à devoir créer leur propre affaire, ne serait-ce qu'à travers des pratiques d'externalisation. On peut donc s'attendre à une diversification croissante des mobiles et des conditions de la création-reprise.

\section{Vers un nouvel entrepreneuriat?}

Au total, on est donc en droit de s'interroger sur la pénétration réelle de l'esprit d'entreprise en France, au-delà de ce qui peut paraitre comme une incantation, une exhortation à changer les mœurs. Mais, paradoxalement, ou dialectiquement, des tendances de fond, moins spectaculaires, impriment des changements en profondeur dans la société française, comme en témoignent divers travaux, lesquels plaident en faveur d'un renouveau de l'esprit d'entreprise, voire d'un nouvel entrepreneuriat.

Ainsi, l'étude réalisée par le Groupe EuroPME, dirigé par l' «équipe Duchéneaut » à l'ESC Rennes, fournit de précieuses informations sur les sociostyles du créateur d'entreprise, en 1998, quitte à tenter d'en dresser un portrait-robot. En fait, l'étude, menée à partir de plus de mille questionnaires, fait apparaître une grande diversité de situations, de comportements, même si des constantes se dégagent, comme la satisfaction globale et le rejet du statut salarial. Il serait trop long de commenter les résultats, fouillés et passionnants. Notons la typologie établie en fin d'étude; les auteurs proposent de retenir deux axes: l'un de proximité ou d'éloignement du milieu de l'entreprise, l'autre d' «état de continuité » ou de rupture. Ils obtiennent quatre catégories : les débutants (proximité et rupture), à raison de $6 \%$, les initiés (proximité-continuité : $25 \%$ ), les matures (éloignementcontinuité : $41 \%$ ) et, enfin, les rebelles (éloignement-rupture : $28 \%$ ).

Ainsi, ce travail fournit un éclairage précieux sur certaines questions, telles que le rôle du milieu d'origine, l'importance de la thèse du département de Shapero, etc. De même, on constate que le monde des «chasseurs » et des «chassés » se partage équitablement. Mais, au total, il est réconfortant de constater que «globalement, la plupart des créateurs affichent une grande motivation et ne souhaitent pas changer de vie. Avec le recul, $89 \%$ déclarent avoir fait le meilleur choix, $83 \%$ seraient très certainement prêts à recommencer, et $15 \%$ peut-être » (p. 71). Ils ne seraient que $8 \%$ à accepter «très certainement » un emploi salarié à revenus équivalents. Cette observation mériterait d'être comparée avec les conclusions tirées de la recherche d'Alain Fayolle sur les ingénieurs.

De même, elle mérite d'être confrontée aux évolutions observées dans un milieu très ambigu en matière d'esprit d'entreprise : l'artisanat. À la fin des années 1980 , nous avions nous-même avancé l'idée d'un «nouvel artisanat », plus entreprenant, moderniste ; un numéro récent des Cahiers de Générations confirme ce 
sentiment. Les auteurs soulignent les transformations en profondeur du milieu des entreprises artisanales, de moins de 10 salariés, englobées de façon croissante dans le monde des TPE. En effet, le poids de la transformation matérielle des produits s'effrite par rapport à celui des services au sein de la chaîne de valeur; de même, le niveau de qualification des artisans s'élève à mesure que de nouvelles technologies pénètrent, et prospèrent, au sein des TPE. Les auteurs plaident pour une transformation en profondeur du statut de l'artisanat : gageons que cette proposition ne sera pas du goût de toutes les institutions consulaires et professionnelles ! Pourtant, le monde de l'artisanat recèle de larges perspectives de développement pour certains métiers, même si, globalement, le nombre d'entreprises ne cesse de diminuer, dans certains métiers, au regard de certaines techniques et dans certaines zones géographiques. Cependant, les rapports entre l'entrepreneuriat et le territoire sont en voie de modifications profondes : nombre de TPE s'installent hors des zones sururbanisées, où les déséconomies d'agglomération, y compris la concurrence intense au sein de districts, deviennent insupportables. L' « encastrement territorial » induit de nouveaux types d'entrepreneurs «entreprenants », comme nous avons tenté de le montrer, dans un numéro spécial de la revue Sciences de la Société (LERASS de Toulouse), dirigé par Jean-Pierre Gilly, paru à l'automne 1999, ainsi que dans la présentation du numéro spécial de la RIPME consacré à la ruralité.

Mais d'autres perspectives entrepreneuriales se font jour, dans des secteurs d'activité qui ne sont pas sans rappeler le nomadisme postmoderne évoqué au début. Ainsi, un sociologue, P. Nicolas-Le Strat, propose une «sociologie du travail artistique », dans laquelle il décrit les conditions de travail du créateur, ou de l'acteur, notamment dans les arts plastiques et les arts du spectacle. Dans le chapitre 6, il décrit cette activité comme «entrepreneuriale » : au demeurant, soit comme intermittent du spectacle, soit comme membre d'une compagnie ou d'une association, révélant l' « entrepreneur individuel », l' « entrepreneur autonome » ou le collaborateur d'une TPE. Ainsi, à côté de la prestation proprement dite, les tâches en amont et en aval occupent une part croissante du temps et exigent une expertise croissante, notamment en matière de communication et d'apprentissage : on retrouve ici la notion de «capital social » chère à Boutillier et Uzunidis.

La même réflexion devrait être menée en ce qui concerne les activités sportives, au sein de véritables « systèmes des sports », pour reprendre l'expression de Paul Yonnet. L'auteur distingue le sport-spectacle, le sport-loisir et le sport de masse. Sur chacun de ces sous-systèmes viennent se greffer une multitude de TPE, souvent fort entreprenantes, qu'elles soient installées en zone urbaine ou rurale. De nouveaux types d'entrepreneurs se mettent en place, qui préfigurent dans une large mesure les activités d'un futur proche, postmoderne. Une attention accrue devrait leur être accordée dans les recherches sur l'entrepreneuriat. 
En conclusion, ces quelques lectures attestent de l'extrême dynamisme de la recherche sur l'entrepreneuriat. On aura remarqué que peu de chercheurs s'interrogent sur les incidences de cette évolution sur la nature même de la petite et moyenne entreprise. De nouvelles voies de recherche méritent donc d'être explorées.

\section{Bibliographie}

BÉRANGER et al. (1998), Rapport sur la formation entrepreneuriale des ingénieurs, Ministère de l'Économie, des Finances et de l'Industrie, octobre, 131 p.

BOUTILLIER, S. et D. UZUNIDIS (1999), La légende de l'entrepreneur. Le capital social ou comment vient l'esprit d'entreprise, Syros, $152 \mathrm{p}$.

Deleuze, G. et F. GuatTARi (1997), Mille plateaux, réédition, Paris, Éditions de Minuit, $645 \mathrm{p}$.

EUROPME (1999), Portait-robot et sociostyles des créateurs d'entreprise en 1998, ESC Rennes, 96 p.

FAYOLLE, A. (1999), L'ingénieur entrepreneur français, contribution à la compréhension des comportements de création et reprise d'entreprise des ingénieurs diplômés, Paris, L'Harmattan, 481 p.

GHOSHAL, S. et C.A. BARTLETT (1998), L'entreprise individualisée. Une nouvelle logique de management, Paris, Maxima, $280 \mathrm{p}$.

HERNANDEZ, E.M. (1999), Le processus entrepreneurial. Vers un modèle stratégique d'entrepreneuriat, Paris, L'Harmattan, $256 \mathrm{p}$.

LE GoFF, J.P. (1999), La barbarie douce. La modernisation aveugle des entreprises et de l'école, Paris, La Découverte, 126 p.

MAFFESOLI, M. (1997), Du nomadisme. Vagabondages initiatiques, Paris, Livre de Poche Biblio Essais, 190 p.

Musso, P. (1999), Saint-Simon et le saint-simonisme, Paris, Presses universitaires de France, collection «Que sais-je ?», no 3468,126 p.

Nicolas-Le StRAT, P. (1998), Une sociologie du travail artistique. Artistes et créativité diffuse, Paris, L'Harmattan, $155 \mathrm{p}$.

NGO-MAI et S. RoCCHIA (1999), «Auto-organisation et connaissance de la firme », Revue d'économie industrielle, $\mathrm{n}^{\circ} 88$, p. 237-256.

OCDE (1998), Stimuler l'esprit d'entreprise, La stratégie de l'OCDE pour l'emploi, 318 p.

POLYNOME (1999), Le risque d'entreprendre, Milan, Les Essentiels, 63 p.

SAMmuT, S. (1998), Jeune entreprise. La phase cruciale du démarrage, Paris, L’Harmattan, $188 \mathrm{p}$.

TORRÈs, O. (1999), Les PME Dominos, Paris, Flammarion, 128 p.

VARII AUCTORES (1999), «Artisans, le pari de l'entreprise individuelle », Les Cahiers de Générations, $\mathrm{n}^{\circ}$ 7, SFG, 95 p. 
Verstraete, T. (1998), «Les dimensions cognitive, praxéologique et structurale de l'organisation de type entrepreneurial », Revue internationale de systémique, vol. 12 , $n^{\circ}$ 4, p. 441-471.

WTterwulghe, R. (1999), La PME. Une entreprise humaine, Paris, De Boeck, 173 p.

YonNET, P. (1998), Systèmes des sports, Paris, Gallimard, Bibliothèque des Sciences Humaines, $254 \mathrm{p}$. 